\title{
Recent X-Ray Laser Characterization Experiments at LLNL
}

R.F. Smith, J. Dunn, J. Nilsen, H. Fiedorowicz, A. Bartnik, V. N. Shlyaptsev

This article was submitted to Optical Society of America Topical Meeting, Palm Springs, CA, October 21-24, 2001

\section{February 26, 2002}

U.S. Department of Energy

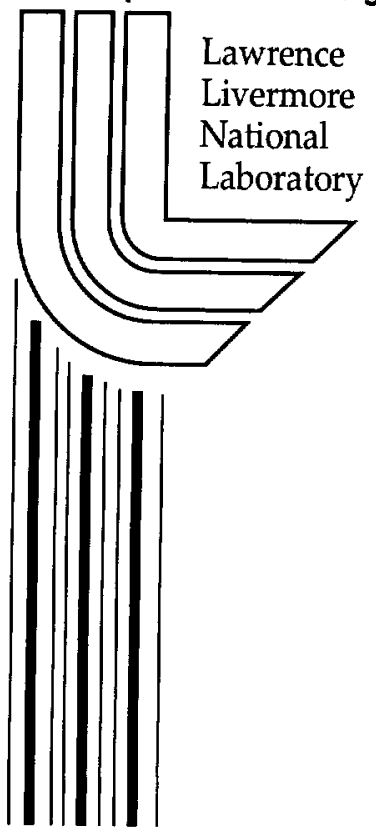




\section{DISCLAIMER}

This document was prepared as an account of work sponsored by an agency of the United States Government. Neither the United States Government nor the University of California nor any of their employees, makes any warranty, express or implied, or assumes any legal liability or responsibility for the accuracy, completeness, or usefulness of any information, apparatus, product, or process disclosed, or represents that its use would not infringe privately owned rights. Reference herein to any specific commercial product, process, or service by trade name, trademark, manufacturer, or otherwise, does not necessarily constitute or imply its endorsement, recommendation, or favoring by the United States Government or the University of California. The views and opinions of authors expressed herein do not necessarily state or reflect those of the United States Government or the University of California, and shall not be used for advertising or product endorsement purposes.

This is a preprint of a paper intended for publication in a journal or proceedings. Since changes may be made before publication, this preprint is made available with the understanding that it will not be cited or reproduced without the permission of the author.

This work was performed under the auspices of the United States Department of Energy by the University of California, Lawrence Livermore National Laboratory under contract No. W-7405-Eng-48.

This report has been reproduced directly from the best available copy.

Available electronically at http: $/ / w w w . d o c . g o v /$ bridge

Available for a processing fee to U.S. Department of Energy

And its contractors in paper from

U.S. Department of Energy

Office of Scientific and Technical Information

P.O. Box 62

Oak Ridge, TN 37831-0062

Telephone: (865) 576-8401

Facsimile: (865) 576-5728

E-mail: reports@adonis.osti.gov

Available for the sale to the public from

U.S. Department of Commerce

National Technical Information Service

5285 Port Royal Road

Springfield, VA 22161

Telephone: (800) 553-6847

Facsimile: (703) 605-6900

E-mail: orders@ntis.fedworld.gov

Online ordering: http://www.ntis.gov/ordering.htm

OR

Lawrence Livermore National Laboratory

Technical Information Department's Digital Library

http://www.llnl.gov/tid/Library.html 


\title{
Recent x-ray laser characterization experiments at LLNL
}

\author{
Raymond F. Smith, James Dunn, Joseph Nilsen, Henryk Fiedorowicz' ${ }^{1}$, Andrzej Bartnik', Vyacheslav N. \\ Shlyaptsev ${ }^{2}$ \\ Lawrence Livermore National Laboratory, Livermore, CA 94550 \\ 'Institute of Optoelectronics. Military University of Technologv, w. Kaliskiego 2, 00-908 Warsan, Poland \\ 2 Dept. of Applied Science, University of Califomia Davis-Livermore, Livermore, CA 94550
}

\begin{abstract}
We report on a series of experiments, using the COMET picosecond facility, designed to characterize and develop different $x$-ray laser sources. This work encompasses collisional pumping of slab and gas puff targets.

(2000 Optical Society of America

OCIS codes: (140.7240) UV, XUV, and X-ray lasers; (340.7440) X-ray imaging
\end{abstract}

\section{Introduction}

In recent years $x$-ray lasers have made rapid progress towards higher efficiency, reduced size, low cost and high repetition rate. These features are scientifically attractive and important for future development of applications that take advantage of the unique characteristics of these sources. Saturated $x$-ray laser operation has been observed at wavelengths in the 20 to $220 \mathrm{eV}$ range with pulse widths between 2 and $800 \mathrm{ps}$. Narrow fractional bandwidth $(\Delta \lambda / \lambda$ $\sim 10^{-4}$ ), conversion efficiencies of $\sim 10^{-6}$, low divergence $(\sim \mathrm{mrad})$ and high saturation intensity $\left(\sim 10^{11} \mathrm{~W} / \mathrm{cm}^{2}\right)$ are characteristic of the $x$-ray laser output which make it the highest brightness single pulse source of $x$-rays currently available.

Collisional $x$-ray lasers typically operate in single/double pass, high gain, amplified spontaneous emission (ASE) mode where the gain region is an extended plasma column. Population inversion is achieved by collisional excitation of the lasing ion by free electrons. In this scheme an optical laser is incident onto a slab target of the lasing material in a line focus geometry. Under the optimal pumping conditions a plasma gain medium column is generated in which the $x$-ray photons which traverse axially undergo preferential amplification. A number of different pumping schemes have been demonstrated. In this paper we describe results using the transient collisional excitation scheme as described by Afanasiev and Shlyaptsev [1]. Two laser pulses are utilized where a long nanosecond pulse at $10^{11}-16^{2} \mathrm{~W} \mathrm{~cm} \mathrm{~cm}^{-2}$ generates the plasma and creates the required closed shell $\mathrm{Ne}$-like or Ni-like ionization conditions. A delay, depending on the initial plasma conditions, is required for plasma cooling and expansion. This is desirable for both optimum pumping and ray propagation along the plasina column. A second much shorter picosecond laser pulse at $10^{14}-10^{15} \mathrm{~W} \mathrm{~cm}$ rapidly generates a transient population inversionThe fast picosecond timescale heating pulse is less than the time it takes for collisional redistribution of the excited levels and allows efficient pumping without perturbing the ionization. Very high $x$-ray laser gains, greater than $100 \mathrm{~cm}^{-1}$, are predicted with the possibility of saturation for target lengths less than $1 \mathrm{~cm}$. High saturation intensities $\left(\sim 10^{1 \mathrm{i}}\right.$ $\left.\mathrm{W} / \mathrm{cm}^{2}\right)$, small source sizes $(50 \times 80 \mu \mathrm{m})$ and narrow angular divergence $(\sim 3 \mathrm{mrad})$ within a $\sim$ ps pulse contribute to the high brightness output [2].

In this paper we describe recent experimental progress at LLNL to characterize the parameters of laser-gas puff soft-X-ray lasers. Near-field and far-field imaging results are presented for the $\mathrm{Ni}$-like Pd X-ray laser.

\section{Experimetal Description}

The experiments were performed on the Compact Multipulse Terawatt (COMET) laser system at LLNL as described previously [3]. This laser, operating at $1054 \mathrm{~nm}$ wavelength, utilizes the technique of chirped pulse amplification to produce three high power beams of nominally $500 \mathrm{fs}$ (compressed) and 600 ps (FWHM) pulse duration with a repetition rate of 1 shot every 4 minutes. For this work, the short pulse was lengthened to $5-6 \mathrm{ps}$ with energy of $4.5-6.5 \mathrm{~J}$ while the long pulse energy was typically $0.5 \mathrm{~J}$ to $4 \mathrm{~J}$. The latter energy was carefully chosen to produce the desired plasma conditions for a particular experiment. For the Ni-like Pd ion X-ray lasers, the peak-to-peak delay between the laser pulses was found to be optimal at $700 \mathrm{ps}$ with the short pulse arriving after the long pulse. These two laser pulses were delivered in a line focus of $1.65 \mathrm{~cm}$ long using a cylindrical lens and an on-axis paraboloid. The long pulse was defocused to a width of $150-175 \mathrm{~m}$ (FWHM) while the short pulse was focused more tightly to $80-120 \mathrm{~m}$ (FWHM). Due to the finite lifetime of the gain relative to the transit time of the $x$-ray laser trough the gain medium it was necessary to employ traveling wave (TW) pumping. 
The TW geometry was implemented before the focusing optics by using a high-reflectivity, $0_{i}$ dielectric-coated reflection echelon consisting of seven flat vertical mirror segments yielding a traveling wave irradiation with a velocity of $c$ [3]. The main diagnostic to monitor the $x$-ray laser was an on-axis 1200 line $\mathrm{mm}^{-1}$ variable-spaced flat-field grating spectrometer coupled to a back-thinned $1024 \times 1024$ (pixel size $24 \times 24 \mathrm{~m}^{2}$ ) charge-coupled device (CCD).

\section{Gas Puff X-ray Laser Experiments}

In this paper we present for the first time a transient gain soft $\mathrm{x}$-ray laser produced by laser irradiation of a gas puff target, instead of a solid [4]. The gas puff target is formed by pulsed injection of gas from a high-pressure solenoid valve through a nozzle in a form of a narrow slit. Soft $\mathrm{x}$-ray lasing with neon-like argon and nickel-like xenon ions was demonstrated 5 years ago using gas puff targets irradiated with $0.5 \mathrm{~ns}$ duration, $400-500 \mathrm{~J}$ energy pulses at large-scale laser facilities [5]. Low gain less than $2 \mathrm{~cm}^{-1}$ was observed in those experiments. While much progress has been made with the capillary discharge $\mathrm{x}$-ray laser technique for Ne-like Ar [6], there is substantial interest in generating a laser-driven gas-puff $x$-ray laser using a tabletop, picosecond laser. In the present experiment we used a combination of a $0.6 \mathrm{~ns}$ prepulse followed by a $6 \mathrm{ps}$ drive pulse, with a total of $10 \mathrm{~J}$, to create soft $\mathrm{x}$-ray lasing in neon-like argon gas puff targets. Fig. 1 shows an on-axis argon spectrum with the strong collisionally excited $3 p^{1} S_{0}$ $\rightarrow 3 s^{1} P_{1}$ laser line at $46.9 \mathrm{~nm}$ and the $3 d^{1} P_{1} \rightarrow 3 p{ }^{1} P_{1}$ laser line at $45.1 \mathrm{~nm}$. The latter has the additional population mechanism of self-photopumping by a resonance line [7]. In Fig. 2 we show the intensity versus plasma column length for the $3 p \rightarrow 3 s$ and $3 d \rightarrow 3 p$ laser lines. Using the Linford formula [8], we estimated a gain coefficient of 10.6 $\mathrm{cm}^{-1}$ for the $3 p \rightarrow 3 s$ line and $10.6 \mathrm{~cm}^{-1}$ for the $3 d \rightarrow 3 p$ line. This corresponds to a gain-length product for the $0.9 \mathrm{~cm}$ long plasma column of 9.6 for each line.
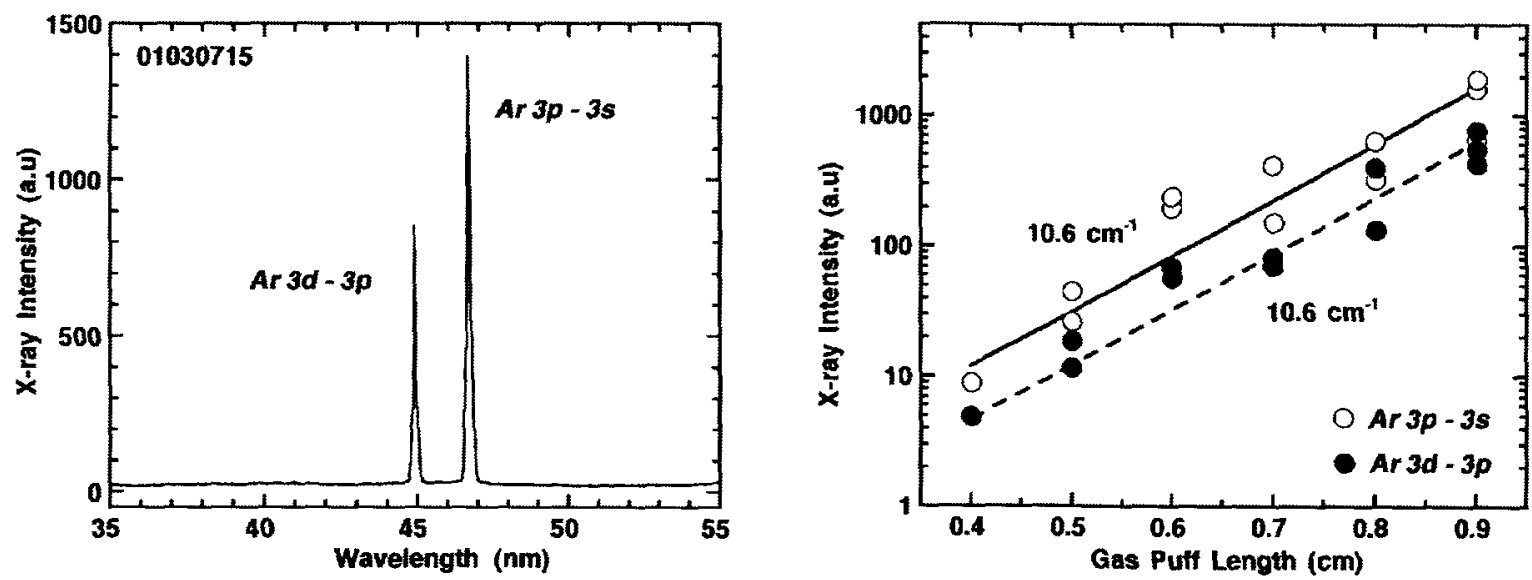

Fig. 1. (Left) On axis spectrum from a $0.9 \mathrm{~cm}$ argon gas puff. The $3 d-\beta$ and the $3 p-3$ lines at $45.1 \mathrm{~nm}$ and $46.9 \mathrm{~nm}$, respectively, are observed.

Fig. 2. (Right) Ne-like Ar $3 d-p$ (closed circles) and $3 p-3$ (open circles) $x$-ray laser intensity as a function of plasma length. Fits to the Linford formula are shown for each X-ray laser line with fitted small-signal gain coefficient of $10.6 \mathrm{~cm}^{-3}$.

\section{Near Field imaging}

The saturated output of the Ni-like $\mathrm{Pd} 4 d \rightarrow 4 p$ x-ray laser line at $14.7 \mathrm{~nm}$ was characterized in order to optimize the beam properties for application experiments (e.g. interferometry) [9]. Flat polished, high-purity Pd slabs were used to generate the $\mathrm{x}$-ray laser medium. Two $\mathrm{x}$-ray mirror optics, $\mathrm{MoC}_{2}: \mathrm{Si}$ multilayers for the $14.7 \mathrm{~nm}$ wavelength, were used to perform near-field imaging. The $0_{\mathrm{i}}$ spherical $\mathrm{x}$-ray mirror with focal length $f=11.75$ $\mathrm{cm}$ was focused to relay image the $\mathrm{x}$-ray laser beam exit pattern via the $45 ;$ flat $x$-ray mirror onto a back-thinned $C C D$ camera. The camera was positioned $248.1 \mathrm{~cm}$ from the $0_{i}$ spherical mirror giving a system magnification of $20.1 x$. The $x$-ray laser beam was double-passed through a filter $(2000$ Lexan $+750 \quad$ Al) placed between the target and the $0_{i}$ mirror. This filter substantially attenuated the $x$-ray laser signal to prevent saturation of the CCD. An additional light tight filter (2925 $\mathrm{Zr}+1050$ polyimide) was placed in front of the CCD camera. The $0 ;$ mirror could be translated to allow the $x$-ray laser beam to be sent to the flat-field spectrometer to 
measure the far-field beam divergence in the horizontal plane. Thus, the near-field pattern and far-field beamdivergence could be measured under similar laser conditions on alternate shots.

Figure 3(a) shows a near-field image of the Pd laser beam; the laser beam is incident from the right in the $z$ direction (horizontal). The aspect ratio of the laser beam is $5 x$ larger in the $y$-direction (vertical) than the horizontal plane. The dimensions of the laser exit pattern are determined in the horizontal plane by the density gradients in the $z$-direction away from the target surface and in the vertical plane by the interaction of the short pulse laser with the pre-formed plasma. Figure 3(b) shows a lineout through the near-field pattern along the $z$-axis. The target surface for this shot is at $z=0$. It can be seen that the centroid of the emission is approximately $70 \mathrm{~m}$ in front of the target surface with a dimension of $30 \mathrm{~m}$ (FWHM). This latter dimension has been measured to be as small as $22 \mathrm{~m}$ on some shots. The foot of the exit aperture extends uniformly away from the target to more than $150 \mathrm{~m}$. The overall dimensions are strongly affected by the initial plasma conditions particularly the density gradients and degree of ionization. Small changes in the long pulse laser intensity e.g. increasing the energy from 1.25 to $1.8 \mathrm{~J}$ results in an exit beam pattern that exhibits more structure further in front of the target as a result of density gradients.

(a)

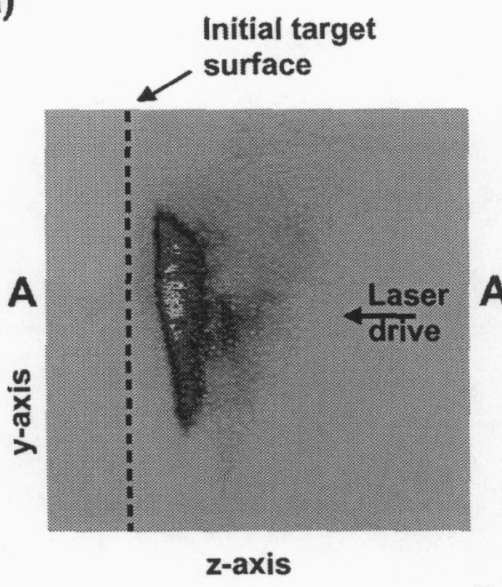

(b)

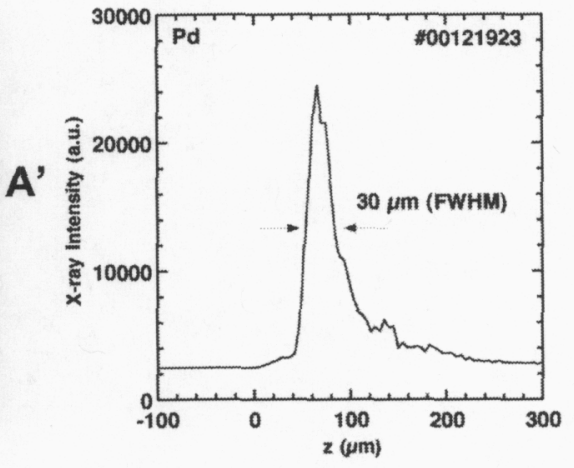

Fig. 3. (a) Near-field image of Ni-like Pd $14.7 \mathrm{~nm}$ x-ray laser beam as it exits the plasma column. The laser drive is incident from the right. The dashed line shows the approximate position of the initial target surface. (b) Lineout through the center of the image marked AA in the $z$-direction away from the target.

\section{References}

1. Yu.V. Afanasiev and V.N. Shlyaptsev, Sov. J. Quant. Electron. 19, pp. 1606-1612 (1989).

2. J.Y. Lin, G.J. Tallents, J. Zhang, A.G. MacPhee, C.L.S. Lewis, D. Neely, J. Nilsen, G.J. Pert, R.M.N. O'Rourke, R. Smith \& E. Wolfrum, Opt. Comm. 158, 55 (1998). ${ }^{\circ}$

3. J. Dunn, Y. Li, A. L. Osterheld, J. Nilsen, J. R. Hunter, and V. N. Shlyaptsev, Phys. Rev. Lett. 84, pp. 4834-4837 (2000); J. Dunn, J. Nilsen, A.L. Osterheld, Y. Li, and V.N. Shlyaptsev, Opt. Lett. 24, 101 (1999).

4. H. Fiedorowicz, A. Bartnick, J. Dunn, R.F. Smith, J. R. Hunter, J. Nilsen, A.L. Osterheld, and V.N. Shlyaptsev, Demonstration of a neonlike argon soft $\mathrm{x}$-ray laser using a picosecond-laser-irradiated gas puff target, Opt. Lett. in press (2001).

5. H. Fiedorowicz, A. Bartnik, Y. Li, P. Lu, and E. E. Fill, Phys. Rev. Lett. 76, 415 (1996); D. Ros et al., Opt. Commun. 153, 368 (1998).

6. B.R. Benware, C.D. Macchietto, C.H. Moreno, and J.J. Rocca, Phys. Rev. Lett. 81, 5804 (1998).

7. J. Nilsen, H. Fiedorowicz, A. Bartnik, Y. Li, P. Lu, and E. E. Fill, Opt. Lett. 21, 408 (1996).

8. G. J. Linford, E. R. Peresini, W. R. Sooy, and M. L. Spaeth, Appl. Opt. 13, 379 (1974).

9. J. Dunn, J. Nilsen, R.F. Smith, A.L. Osterheld, V.N. Shlyaptsev, J. Filevich, J.J. Rocca, and M.C. Marconi, Recent X-ray Laser Experiments on the COMET Facility, Presented at Soft X-ray Lasers and Applications IV, SPIE International Symposium on Optical Science and Technology, San Diego, California, 29 July - 3 August 2001.

\section{Acknowledgements}

This work was performed under the auspices of the U.S. Dept. of Energy by the University of California Lawrence Livermore National Laboratory under Contract No. W-7405-Eng-48 and in part by grant No. 2 P03B 09316 of the State Committee for Scientific Research of Poland. 